\title{
Missense mutations in SARS-CoV2 genomes from Indian patients
}

\author{
Sk. Sarif Hassan ${ }^{\mathrm{a}, *}$, Pabitra Pal Choudhury ${ }^{\mathrm{b}}$, Bidyut Roy ${ }^{\mathrm{c}}$, Siddhartha Sankar Jana ${ }^{\mathrm{d}}$ \\ ${ }^{a}$ Department of Mathematics, Pingla Thana Mahavidyalaya, Maligram 721140, India \\ ${ }^{b}$ Applied Statistics Unit, Indian Statistical Institute, Kolkata 700108, West Bengal, India \\ ${ }^{c}$ Human Genetics Unit, Indian Statistical Institute, Kolkata 700108, West Bengal, India \\ ${ }^{d}$ School of Biological Sciences, Indian Association for the Cultivation of Science, West Bengal, 700032, India.
}

\begin{abstract}
As on May 24, 2020, in the NCBI database, there are complete genome sequences of SARS-CoV2 from 128 Indian patients. In this present study variations among these genomes based on missense mutations are accounted and thereby some key findings with some hypotheses are made. These mutations across various genes of these genomes show a wide genetic variations in sequence and rapid evolution of SARS-CoV2 virus.
\end{abstract}

Keywords: Indian SARS-CoV2 genomes; Missense mutations; Genetic variations.

\section{The study}

The pandemic of COVID-19, caused by the RNA coronavirus SARS-CoV2 has spread over 200 countries and infected millions of people worldwide [1, 2]. Generally, viral evolution is governed by the nucleotide mutations. Rate of mutation of RNA viruses is much higher than that of their hosts [3, 4]. The rapid spread of COVID-19 across various countries opens an intriguing question whether the variations in genome sequences of the novel coronavirus is contributing to the variation of infection and mortality rate[5]. This was suggested by the work on genetic diversity and evolution of SARS-CoV2 by Tung Phan [6]. Having this motivation, with the reference to the Wuhan based genome NC_045512, missense mutations were identified in the SARS-CoV2 genomes of the COVID-19 affected Indian patients. Genome sequences from 128 Indian patients are available in the NCBI database. A comprehensive list with other associated details are presented in the Table 1. It was noted that most of these 128 patients were infected in Europe and USA and came back to India. Some of them were infected from Indian patients.

\footnotetext{
* Corresponding author

Email addresses: sarimif@gmail.com (Sk. Sarif Hassan), pabitrapalchoudhury@gmail.com (Pabitra Pal Choudhury), broy@isical.ac.in (Bidyut Roy), bcssj@iacs.res.in (Siddhartha Sankar Jana)
} 
Table 1: List of 128 SARS-CoV2 genomes of Indian origin with their respective locations and date of collection of the sample

\begin{tabular}{|c|c|c|c|c|c|c|c|c|}
\hline Accession & Geo_Location & Collection_Date & Accession & Geo_Location & Collection_Date & Accession & Geo_Location & Collection_Date \\
\hline MT509494 & Vadodara & 2020-05-03 & MT496981 & Ahmedabad & 2020-05-03 & MT467252 & Ahmedabad & 2020-04-29 \\
\hline MT509495 & Kodinar & $2020-05-10$ & MT496982 & Ahmedabad & 2020-05-03 & MT 467253 & Ahmedabad & 2020-04-29 \\
\hline MT509496 & Botad & 2020-05-09 & MT496983 & Ahmedabad & 2020-05-05 & MT 467254 & Ahmedabad & 2020-04-29 \\
\hline MT509497 & Una & $2020-05-11$ & MT496984 & Ahmedabad & 2020-05-03 & MT467255 & Ahmedabad & $2020-04-30$ \\
\hline MT509499 & Jamnagar & 2020-05-07 & MT496986 & Ahmedabad & 2020-05-05 & MT 467257 & Ahmedabad & $2020-04-30$ \\
\hline MT509500 & Dahod & 2020-05-03 & MT 496987 & Ahmedabad & 2020-05-04 & MT 467258 & Ahmedabad & $2020-04-29$ \\
\hline MT509501 & Jamnagar & 2020-05-06 & MT496988 & Ahmedabad & $2020-05-03$ & MT467259 & Prantij & 2020-05-02 \\
\hline MT509502 & Jamnagar & $2020-05-10$ & MT496989 & Ahmedabad & 2020-05-03 & MT 467260 & Prantij & 2020-05-02 \\
\hline MT509503 & Junagadh & 2020-05-09 & MT496990 & Gandhinagar & 2020-04-29 & MT467261 & Modasa & 2020-05-04 \\
\hline MT509504 & Rajkot & 2020-05-06 & MT496991 & Gandhinagar & $2020-04-27$ & MT467262 & Modasa & 2020-05-04 \\
\hline MT509506 & Rajkot & $2020-04-27$ & MT496993 & Gandhinagar & $2020-04-29$ & MT 457402 & Hyderabad & 2020-03-24 \\
\hline MT509507 & Una & $2020-05-10$ & MT496994 & Gandhinagar & 2020-04-29 & MT 457403 & Hyderabad & $2020-03-25$ \\
\hline MT509508 & Jamnagar & 2020-05-05 & MT496995 & Gandhinagar & 2020-04-29 & MT 451874 & Surat & $2020-04-24$ \\
\hline MT509509 & Ahmedabad & 2020-04-11 & MT496996 & Gandhinagar & 2020-05-02 & MT 451876 & Surat & $2020-04-26$ \\
\hline MT509510 & Una & 2020-05-10 & MT496997 & Gandhinagar & $2020-04-29$ & MT 451877 & Surat & $2020-04-26$ \\
\hline MT509511 & Rajkot & $2020-04-28$ & MT 483553 & Modasa & $2020-05-05$ & MT 451878 & Surat & $2020-04-27$ \\
\hline MT509512 & Dahod & 2020-05-01 & MT483554 & Modasa & 2020-05-05 & MT 451880 & Surat & $2020-04-26$ \\
\hline MT481895 & Ahmedabad & $2020-05-03$ & MT 483555 & Modasa & 2020-05-05 & MT 451881 & Ahmedabad & $2020-04-26$ \\
\hline MT481896 & Ahmedabad & $2020-05-03$ & MT483556 & Modasa & 2020-05-05 & MT 451882 & Ahmedabad & $2020-04-26$ \\
\hline MT481897 & Modasa & 2020-05-05 & MT483557 & Modasa & 2020-05-05 & MT 451883 & Ahmedabad & $2020-04-26$ \\
\hline MT481898 & Himatnagar & $2020-05-05$ & MT 483558 & Modasa & $2020-05-05$ & MT 451884 & Ahmedabad & $2020-04-26$ \\
\hline MT481899 & Modasa & 2020-05-05 & MT 483559 & Prantij & 2020-05-05 & MT 451885 & Ahmedabad & $2020-04-26$ \\
\hline MT481902 & Dahegam & $2020-05-03$ & MT477885 & India* & $2020-03-25$ & MT 451888 & Ahmedabad & $2020-04-26$ \\
\hline MT481903 & Dahegam & $2020-05-03$ & MT467237 & Ahmedabad & $2020-04-26$ & MT 451889 & Ahmedabad & $2020-04-26$ \\
\hline MT481904 & Gandhinagar & $2020-04-25$ & MT467238 & Ahmedabad & 2020-04-29 & MT451890 & Ahmedabad & $2020-04-26$ \\
\hline MT481905 & Gandhinagar & 2020-05-02 & MT467239 & Ahmedabad & $2020-04-26$ & MT 435079 & Ahmedabad & $2020-04-13$ \\
\hline MT481906 & Gandhinagar & $2020-04-27$ & MT467240 & Ahmedabad & $2020-04-30$ & MT 435080 & Ahmedabad & $2020-04-13$ \\
\hline MT481907 & Gandhinagar & 2020-04-26 & MT467241 & Ahmedabad & $2020-04-26$ & MT435081 & Ahmedabad & $2020-04-13$ \\
\hline MT481908 & Mansa & $2020-04-28$ & MT 467242 & Ahmedabad & 2020-04-29 & MT 435082 & Ahmedabad & $2020-04-13$ \\
\hline MT481909 & Modasa & $2020-05-05$ & MT 467243 & Ahmedabad & $2020-04-30$ & MT 435083 & Ahmedabad & 2020-04-07 \\
\hline MT496972 & Ahmedabad & 2020-04-29 & MT 467244 & Ahmedabad & $2020-04-30$ & MT 435084 & Ahmedabad & $2020-04-14$ \\
\hline MT496973 & Ahmedabad & $2020-04-29$ & MT467245 & Ahmedabad & $2020-04-29$ & MT435085 & Gandhinagar & $2020-04-22$ \\
\hline MT496974 & Ahmedabad & 2020-04-29 & MT467246 & Ahmedabad & 2020-04-29 & MT435086 & Mansa & $2020-04-21$ \\
\hline MT496975 & Ahmedabad & 2020-04-29 & MT 467247 & Ahmedabad & 2020-04-29 & MT415320 & India* & 2020-03-01 \\
\hline MT496976 & Ahmedabad & 2020-05-03 & MT467248 & Ahmedabad & 2020-04-29 & MT415321 & India* & 2020-03-11 \\
\hline MT496977 & Ahmedabad & 2020-05-03 & MT467249 & Ahmedabad & 2020-04-29 & MT415322 & India* & $2020-03-16$ \\
\hline MT496979 & Ahmedabad & 2020-05-05 & MT 467250 & Ahmedabad & 2020-04-29 & MT415323 & India* & 2020-03-20 \\
\hline \multirow[t]{3}{*}{ MT 496980} & Ahmedabad & $2020-05-03$ & MT 467251 & Ahmedabad & $2020-04-29$ & MT358637 & Rajkot & $2020-04-05$ \\
\hline & & & & & & MT012098 & Kerala State & $2020-01-27$ \\
\hline & & & & & & MT050493 & Kerala State & $2020-01-31$ \\
\hline
\end{tabular}

* No specific name of the state/city of India is mentioned in the NCBI database.

The following Tables 2, 3, 4 and 5 adumbrate all the missense mutations along with silent mutations across the 128 genomes of the Indian COVID-19 affected patients.

Mutations of an amino acid $A_{1}$ to an amino acid $A_{2}$ is denoted by $A_{1}(l) A_{2}$ where $l$ denotes location in the reference amino acid sequence. The third column of the Table 2, 3, 4 and 5 describe the regions where the mutations occurred. Here, the protein names are mentioned with the total number of mutations in the parenthesis. 


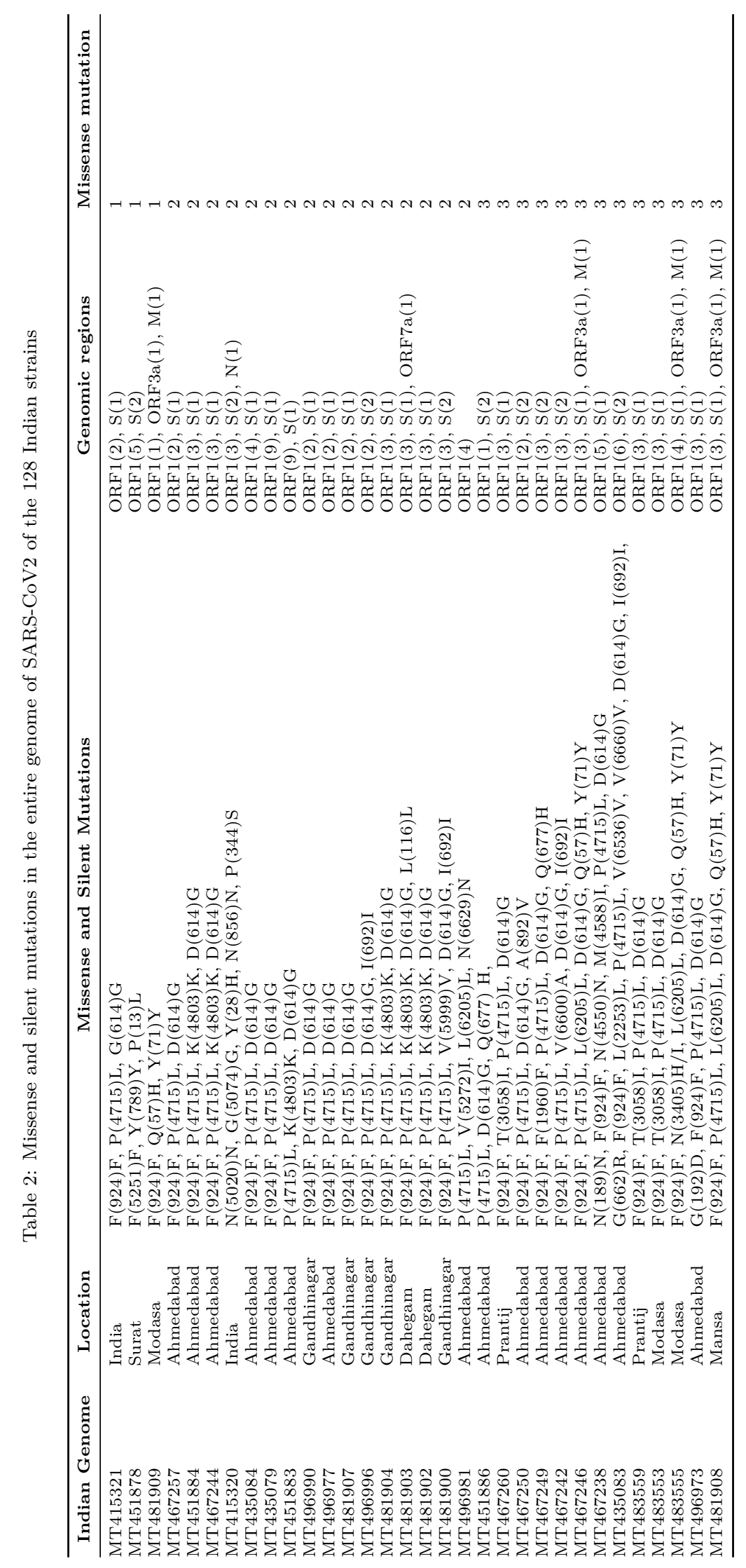




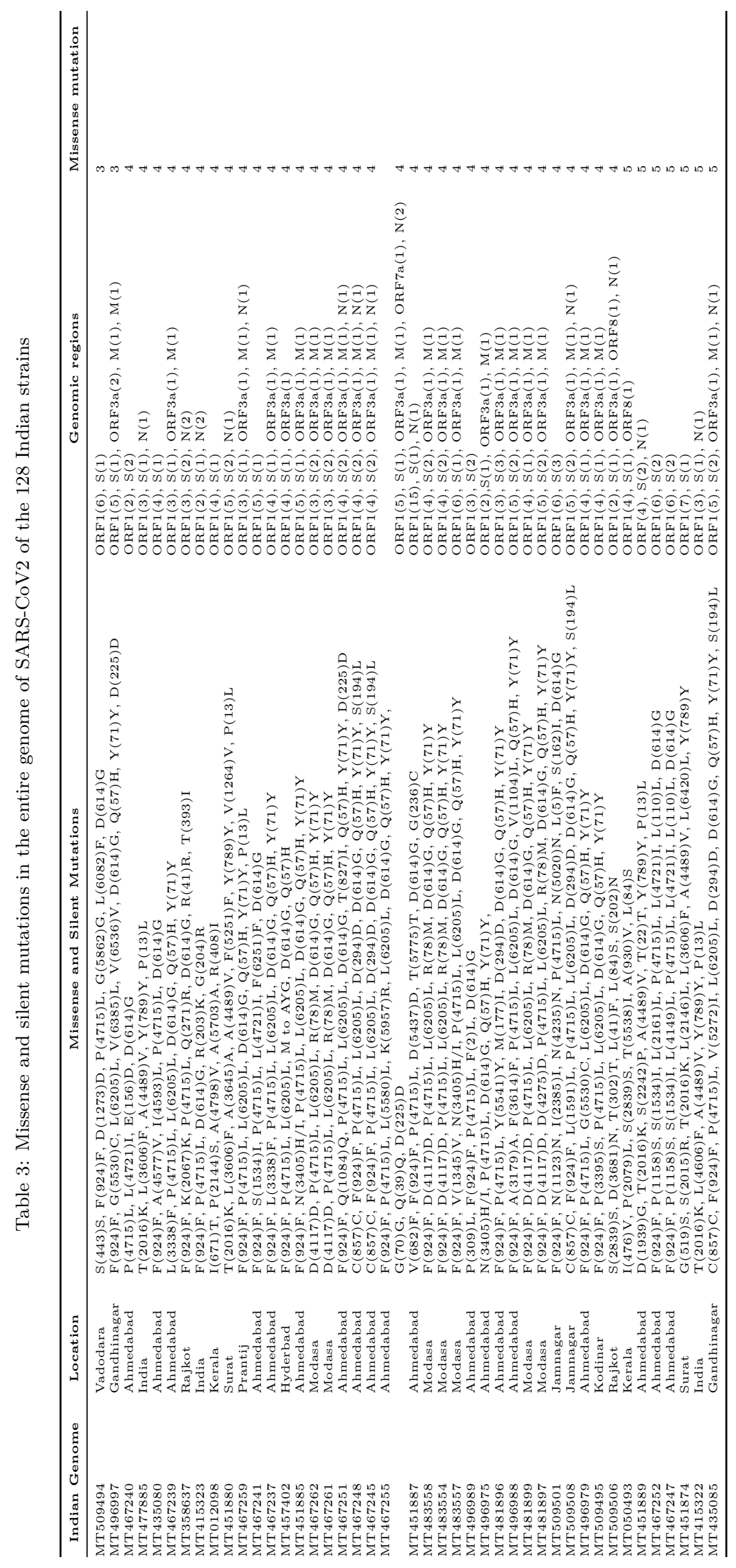




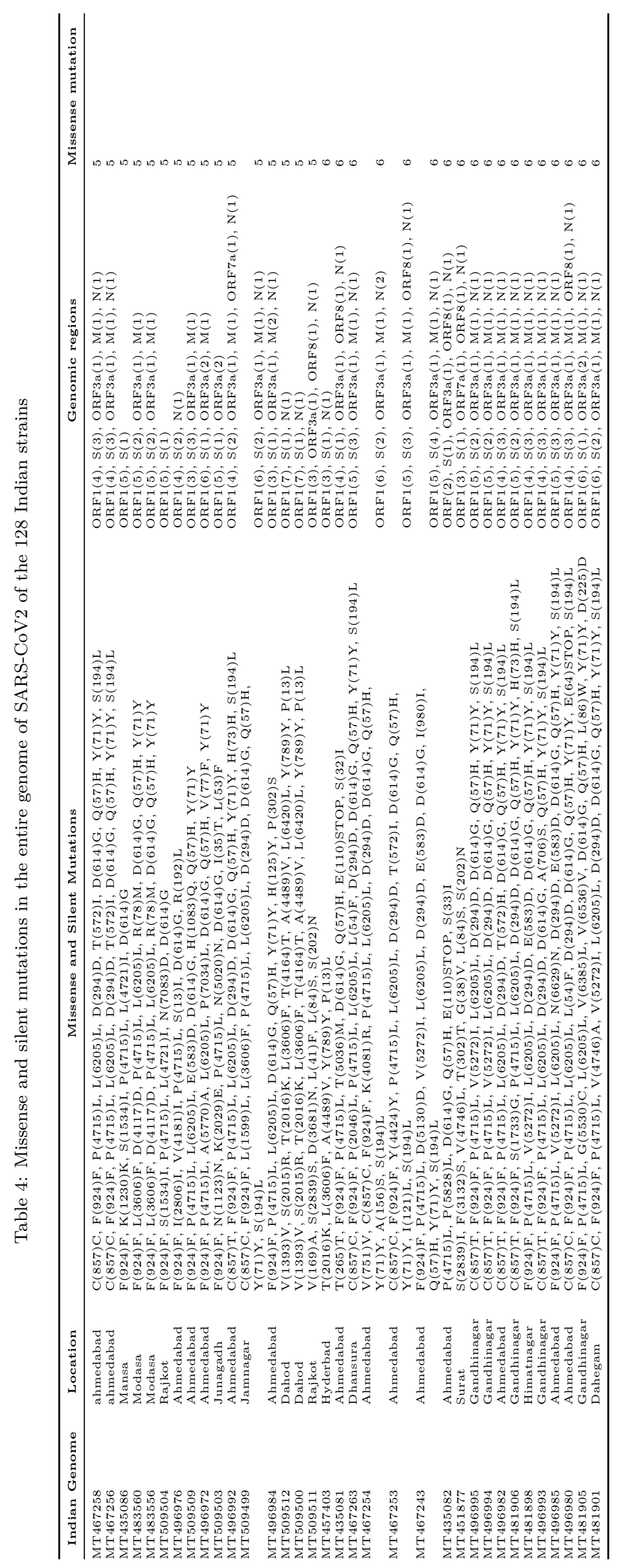




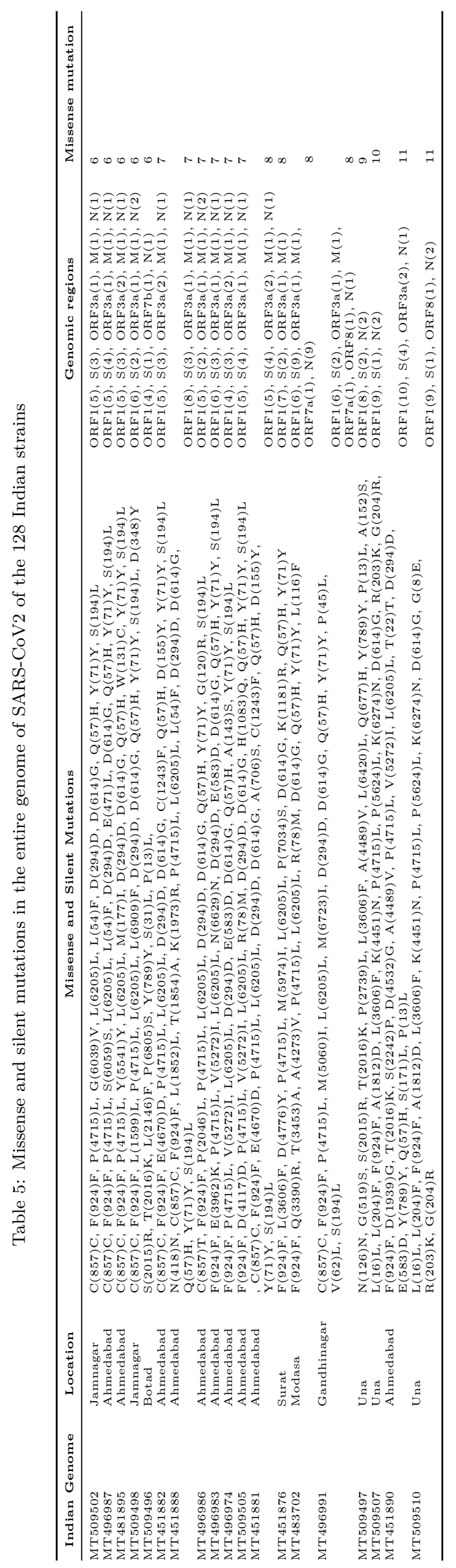




\subsection{Findings from the Table 2, 3, 4 and 5}

There are several synonymous mutations in the different proteins across the genomes of SARS-CoV2 of the Indian patients. Some of the conclusive inferences as well as hypotheses are drawn, based on the missense mutations as presented in the Table 2, 3, 4 and 5 as follows:

- Maximum number of missense mutations were found to be 11 which occurred in two genomes MT451890 (Ahmedabad) and MT509510(Una). Note that the only common missense mutations among these 11 mutations is $\mathrm{P}(4715) \mathrm{L}$. Clearly, the source of infection is different in these two patients.

- From Una, MT509507 genome has ten different missense mutations that are significantly different from others, which is depicted in the Table 5. So the patient carrying the SARS-CoV2 genome MT509507 has a distinct origin of infection.

- No mutation was observed in the ORF6, E, ORF10 proteins of the SARS-CoV2 genomes of the 128 strains. It is worth mentioning that among 128 genomes, MT509496 is the only genome where protein ORF7b possesses a missense mutation $\mathrm{S}(31) \mathrm{L}$.

- The six genomes MT451882, MT451888, MT496986, MT496983, MT496974 and MT509505 from Ahmedabad possess seven missense mutations among which only three are commons viz. $\mathrm{P}(4715) \mathrm{L}, \mathrm{Q}(57) \mathrm{H}$ and S(194)L in the proteins ORF1, ORF3a and N respectively.

- There are six identical missense mutations across the genomes MT496995 and MT496994 from Gandhinagar which imply their same source of infection of COVID-19. Similarly, two genome sequences MT481898(Himatnagar) and MT496985(Ahemedabad), had six identical missense mutations and they are $\mathrm{P}(4715) \mathrm{L}, \mathrm{V}(5272) \mathrm{I}, \mathrm{E}(583) \mathrm{D}, \mathrm{D}(614) \mathrm{G}, \mathrm{Q}(57) \mathrm{H}$ and $\mathrm{S}(194) \mathrm{L}$ in the proteins ORF1, ORF1, S, S, ORF3a and N, respectively. So, these two patients had infection of same COVID-19.

- Each pair of genomes $\{M T 467252, M T 467247\}$ and $\{M T 467258, M T 467256\}$ from Ahmedabad have five identical missense mutations in across various proteins.

- The pairs of genomes $\{M T 483558, M T 483554\}$ and $\{M T 467262, M T 467261\}$ from Modasa hold four identical (pairwise) mutations in ORF1, S, ORF3a and M proteins.

- Except MT509503(Junagadh), all SARS-CoV2 genomes from the Indian patients had mutations at $\mathrm{Q}(57) \mathrm{H}$ and $\mathrm{Y}(71) \mathrm{Y}$ of the protein ORF3a and $\mathrm{M}$ respectively, apart from other mutations.

- There are 13 genomes viz. MT451878, MT451883, MT451886, MT467240, MT467239, MT012098, MT451880, MT467262, MT467261, MT050493, MT451889, MT451874 and MT435082 where the missense mutations are restricted to the structural (except E) and non-structural proteins. 


\section{Conclusions}

Several missense mutations over these 128 SARS-CoV2 genomes of the Indian patients from diverse geolocations show the wide genetic variations. Within a very small time frame, the virus evolved rapidly. Quite a few patients had identical mutations indicating infection received from neighbouring or family patients. It will be interesting to study how many of these variations in sequence in viruses were carried by the patients from outside India or evolved within India. Whether the missense mutations over the 128 genomes lead to different functions of the proteins, is certainly the next question which is to be investigated by further studies. Although the clinical significance of the observed mutations is not readily available, our findings in Indian patients lay the ground work for India to understand the impact of SARS-CoV2 mutations on disease severity, host immune response, vaccine development and serological response.

\section{Author Contributions}

SH, PPC, SSJ conceived the problem. SH determined the mutations. SH, PPC, BR and SSJ analysed the data and result. SH wrote the initial draft which was checked and edited by all other authors to generate the final version.

\section{Conflict of Interests}

The authors do not have any conflicts of interest to declare.

\section{References}

[1] T. Singhal, A review of coronavirus disease-2019 (covid-19), The Indian Journal of Pediatrics (2020) 1-6.

[2] P. Stefanelli, G. Faggioni, A. L. Presti, S. Fiore, A. Marchi, E. Benedetti, C. Fabiani, A. Anselmo, A. Ciammaruconi, A. Fortunato, et al., Whole genome and phylogenetic analysis of two sars-cov-2 strains isolated in italy in january and february 2020: additional clues on multiple introductions and further circulation in europe, Eurosurveillance 25 (13) (2020) 2000305.

[3] J. Cui, F. Li, Z.-L. Shi, Origin and evolution of pathogenic coronaviruses, Nature reviews Microbiology 17 (3) (2019) 181-192.

[4] M. Pachetti, B. Marini, F. Benedetti, F. Giudici, E. Mauro, P. Storici, C. Masciovecchio, S. Angeletti, M. Ciccozzi, R. C. Gallo, et al., Emerging sars-cov-2 mutation hot spots include a novel rna-dependent-rna polymerase variant, Journal of Translational Medicine 18 (2020) 1-9.

[5] F. Yu, L. Du, D. M. Ojcius, C. Pan, S. Jiang, Measures for diagnosing and treating infections by a novel coronavirus responsible for a pneumonia outbreak originating in wuhan, china, Microbes and infection (2020).

[6] T. Phan, Genetic diversity and evolution of sars-cov-2, Infection, Genetics and Evolution 81 (2020) 104260. 